\title{
LARGE SCALE INTEGRATED 3D MICROFLUIDIC NETWORKS THROUGH HIGH YIELD FABRICATION OF VERTICAL VIAS IN PDMS
}

\author{
C.F. Carlborg, K.T. Haraldsson, M. Cornaglia, G. Stemme, and W. van der Wijngaart \\ KTH - Royal Institute of Technology, Stockholm, Sweden
}

\begin{abstract}
This paper introduces a robust, high yield, single-step fabrication method for creating densely spaced, miniaturized out-of-plane fluidic interconnecting channels (=vias) in standard poly(dimethylsiloxane) PDMS. Unblocked vias are essential for creating 3D microfluidic networks. Previously reported methods either had low yield, because of residual membranes covering the vias after polymerization, or required complicated extra steps to remove the blocking membranes.

In contrast, our method prevents the formation of residual membranes by inhibition of the polymerization on top of the protuding mold features defining the vias locations. In addition to providing unblocked vias, the inhibition also leaves a flat partially cured, sticky top surface that adheres well to other surfaces and allows self-sealing stacking of several PDMS layers. We demonstrate the new method by manufacturing a densely perforated PDMS membrane and a large scale integrated (LSI) 3D PDMS microfluidic channel network. Our method enables batch manufacturing of complex fluidic devices by speeding up and simplifying the fabrication of complex microfluidic components in standard PDMS.
\end{abstract}

\section{INTRODUCTION}

The manipulation of multiple liquids in microfluidic systems often requires $3 \mathrm{D}$ channel layouts with under- and overpasses to allow the liquids to cross without mixing. Three dimensional channel networks fabricated using planar soft replica molding, must use at least two microfluidic layers with well-defined vias. Most lab-on-a-chip devices are made from PDMS because of its attractive mechanical, chemical and physical properties. It is therefore essential to develop a method using unmodified PDMS for reliably creating multiple vias to enable large scale integration (LSI) of 3D microfluidic networks.

To minimize the number of aligning and bonding steps, the most efficient approach uses a dual-level mold [1], where the lower mold level defines the lower channels, and the top mold level defines the vias (Fig $1 \mathrm{~A}$ ). The PDMS layer is formed by thermally curing the prepolymer pressed between the mold and a top cover [2] (Fig 1 B). The resulting layer with both channels and vias is aligned and bonded to a second PDMS channel layer, fabricated by a separate mold. This approach promised true miniaturization and large scale integration. However, the PDMS prepolymer tends to cover the protruding vias mold features with a thin blocking layer, even at high clamping pressure, and causes residual PDMS membranes in the vias and thus very low fabrication yield (Fig 1 C) [3]. Previous attempts include removing the residue by mechanically squeezing [1-3] or blowing away [4] the prepolymer residue, or by rupturing the polymerized residue [5], however these are limited in their reproducibility and/or yield.

We previously demonstrated inhibiting the uvpolymerization of epoxy-terminated PDMS on top of a single-level mold, and subsequent flushing away of the unpolymerized residual layer (Fig 1 D) [6]. However, this method required non-standard PDMS with altered mechanical properties, not suited for labon-a-chip devices.

In the current work, we introduce an uncomplicated, single-step method to prevent the local polymerization of standard PDMS by inhibition of its platinum catalyst to achieve residual free vertical vias over large areas.
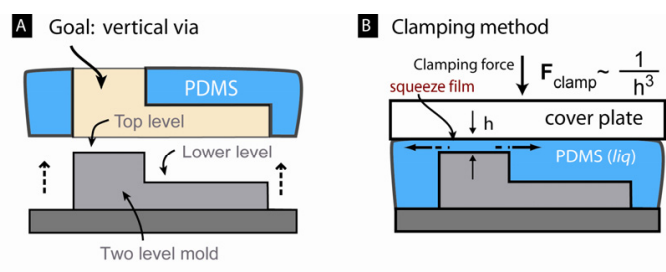

C Problem with clamping method
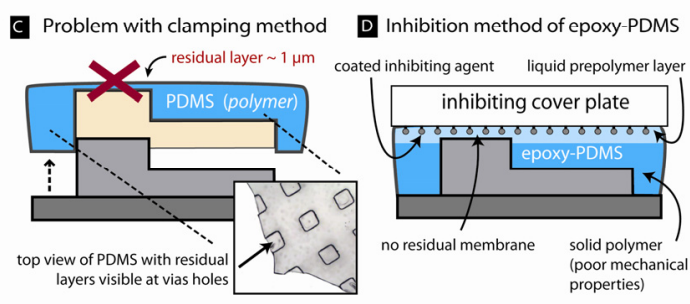

Figure 1 : (A) Muti-liquid 3D microfluidic devices require vertical vias (interconnects) between fluidic layers. (B) Previous method to remove the residual PDMS pre-polymer by clamping is difficult due to the squeeze film effect and often leaves blocked vias (C) that needs to be opened manually. (D) We previously demonstrated membrane free vias by inhibiting the polymerization of an epoxy modified PDMS. 
A

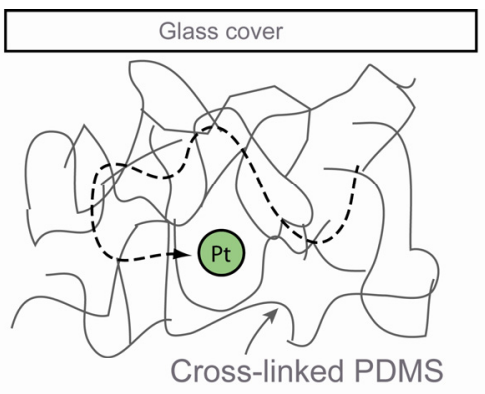

B

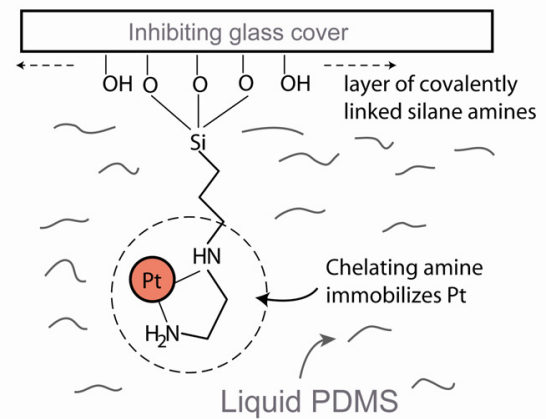

Figure 2: Illustration of the inhibition mechanism. In (A) without the chelating agent the Pt catalyst is initially mobile and can cure the prepolymer. In (B) the monolayer of chelating amines immobilizes the Pt and inhibits polymerization reaction, leaving a liquid prepolymer at the protruding mold features.

\section{INHIBITION MECHANISM}

The standard PDMS (Sylgard 184, Dow Corning, USA) curing process relies on platinum catalyzed hydrosilylation crosslinking reactions between silicon hydride and vinylsiloxane groups. Platinum catalysts are easily deactivated by amines, which bind to the free platinum atom and prevent it from catalyzing the cross linking. To deactivate the platinum only in a thin top layer, we use a chelating organosilane. It can be covalently bonded to a inhibiting glass or silicon surface and that has documented high ability to capture and deactivate the platinum catalyst [7]. When a glass cover coated with the chelating agent is clamped on top of the PDMS prepolymer, it will deactivate platinum at close to the inhibiting glass cover and thus, during the thermal curing process, the top layer between the inhibiting glass cover and the protruding mold features will remain uncured while the bulk is unaffected by the inhibition (Fig 2).

\section{FABRICATION}

\section{Inhibiting glass cover}

Inhibiting covers are first fabricated by immersing glass slides in a solution of the silanized chelated compound aminoethylaminopropyltrimethoxysilane (AEAPS) in methanol, and then baked in the oven to covalently linking the silane groups to the glass. They are then ready for use and can be stored for several weeks with maintained functionality.

\section{Membrane free vias}

Standard PDMS prepolymer is poured on a dual level SU-8 mold, pretreated with Teflon AFTM (Figure 3 A). Next an inhibiting glass cover, coated with the

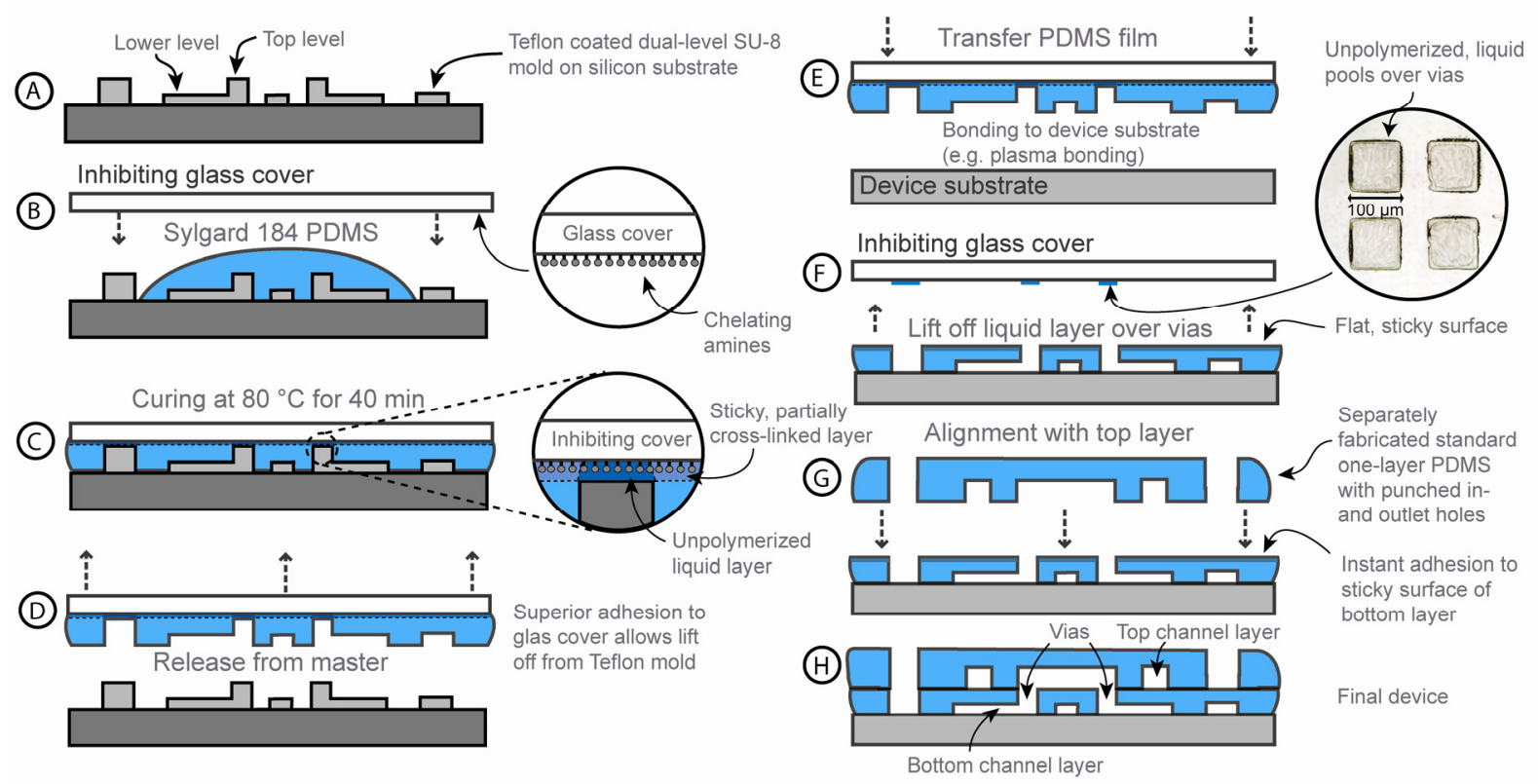

Figure 3: PDMS is poured on a dual layer mold (A), a glass cover coated with the inhibitor is clamped on (B). After curing in oven (C) the glass cover is released with the PDMS attached because the partially cross-linked top layer stick preferentially to glass rather than to the Teflon coated mold (D). The PDMS layer is transferred to the device substrate, bonded $(E)$ and the glass cover is removed, taking with it the unpolymerized layer and leaving a flat sticky surface $(F)$. Finally, additional PDMS layers is aligned and sealed by bringing into contact 
chelating amine, is lightly clamped on top (B). To avoid bubbles, the prepolymer is degassed in vacuum before thermal curing (30 min @ $80{ }^{\circ} \mathrm{C}$ ) in oven. After the curing, a thin layer of unpolymerized PDMS remains above the protruding vias features where all $\mathrm{Pt}$ has been depleted. Outside the via features, the inhibited surface is sticky and only partially crosslinked while the bulk is fully cross-linked (C). When demolding, the sticky surface of the partially cured PDMS surface make the polymer film stick preferentially to the inhibiting glass cover rather than to the Teflon-coated mold (D), and the PDMS layer can be lifted of with the inhibiting glass cover as a carrier. This simplifies the alignment and bonding of the fluidic layer to its final device substrate (E). After the PDMS film has been aligned and bonded to its substrate, for example by using plasma treatment, the inhibiting glass cover can be removed by gently shearing it off, taking with it the unpolymerized liquid PDMS on top of the protruding vias features (F) and leaving a flat, sticky surface with well defined vias without residual membranes. Finally, a top channel layer, fabricated using standard PDMS microreplication, can be aligned with the bottom layer (G) and upon contact immediately seals with the sticky surface of the bottom layer $(\mathrm{H})$. Several PDMS layers can be stacked like this without additional bonding. If desired, the sticky inhibited surface can also directly adhere to various other substrates, such as glass and silicon, creating PDMS stickers.

\section{Demonstrator devices}

To demonstrate the high yield of the inhibition method, we fabricated a $50 \mu \mathrm{m}$ thick perforated PDMS membrane $\left(25 \mathrm{~mm}^{2}\right)$ containing 900 circular holes, $100 \mu \mathrm{m}$ in diameter, with $150 \mu \mathrm{m}$ pitch and a $100 \%$ yield of unblocked vias (Fig 4 ).

To demonstrate the feasibility to build larger 3D devices we also fabricated a 3D "basket-weave" microchannel structure $\left(2 \times 2 \mathrm{~cm}^{2}\right)$ consisting of two layer of PDMS with two sets of 7 parallel channels passing consecutively over and underneath each other, using 64 vertical vias, without the fluids contacting each other (Fig $5 \mathrm{~A}$, C). The channels were filled with coloured liquids to demonstrate the integrity of the channels and the leak tight adhesion between the two PDMS layers (Fig 5).

The adhesive strength between the bottom and top layer was measured to 4 bar (limit of our equipment) by blister test [8].

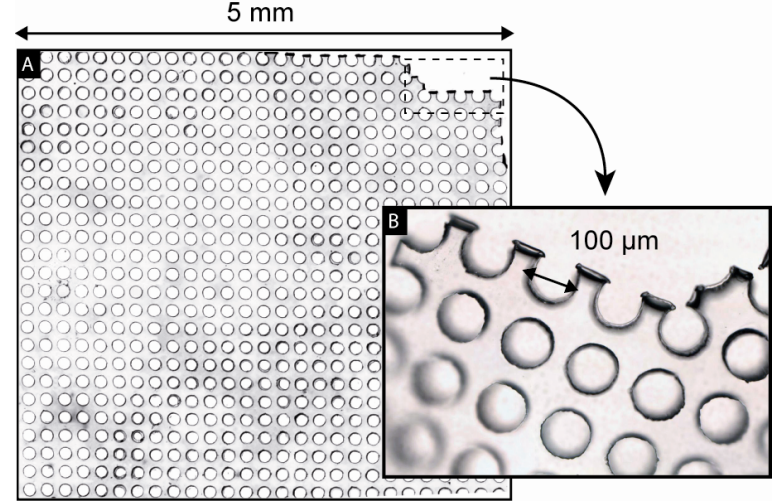

Figure 4: (A) Photograph of a $50 \mu \mathrm{m}$ thick $25 \mathrm{~mm}^{2}$ PDMS layer with 900 trough holes $100 \mu \mathrm{m}$ in diameter. The method was shown to reliable produce thin perforated PDMS layers with $100 \%$ yield over a large area. (B) Zoom-in of the smooth inhibited surface with the trough holes.

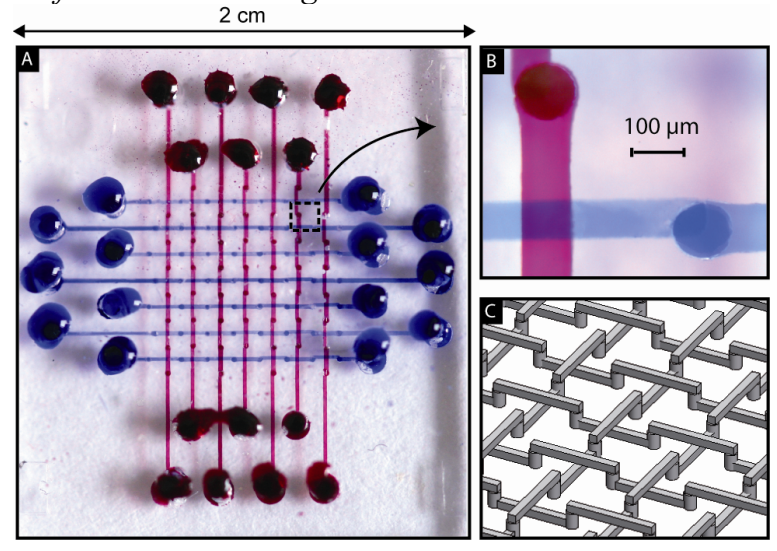

Figure 5: (A) Photograph of a fabricated 3D "basket weave" channel structure in PDMS with channels crossing under and over each other. (B) The channel width in both layers is $100 \mu \mathrm{m}$, the circular vertical interconnects are $120 \mu \mathrm{m}$ in diameter and the top level thickness is $60 \mu \mathrm{m}$. (C) The actual "basket weave structure" shown for clarity as a 3D sketch.

\section{DISCUSSION}

The demonstrated new method provides a number of advantages over previous methods. Firstly, it allows reliable creation of membrane free vias in one single step in standard PDMS. The unblocked vias yield is higher than pure mechanical clamping and it is more robust and batch adapted than tearing off the membranes or blowing away the prepolymer from the protruding vias features. Secondly, the partially crosslinked, sticky, inhibited surface adheres well to PDMS and other materials. This is advantageous when stacking several layers of PDMS to create 3D structures. Unlike plasma bond the sticky surface results in an adhesive and does not form a covalent bond. 


\section{CONCLUSION}

This paper presents an uncomplicated single-step method for the high yield fabrication of PDMS layers with vertical vias and its use in manufacturing of large scale integrated 3D microfluidic network, without modifying the mechanical, physical or chemical material properties. The method is reliable and reproducible, and unlike previous methods, it has a high yield over a large area and is suited for manufacturing of LSI microfluidic networks.

\section{REFERENCES}

[1] J.R. Anderson et al, Anal. Chem, 72 (2000), pp. 3158-3164

[2] B.H. Jo et al., J. Microelectromech. Syst., 9 (2000), pp. 76-81

[3] D.T. Chiu et al., PNAS, 97 (1999), pp. 2408-2413

[4] J.H, Kang et al, J.Micromech. Microeng., 19 (2009), pp. 045027

[5] U. Klotter et al, Proc. IEEE MEMS '04, Maastricht, pp. 745-748

[6] C.F. Carlborg et al. Proc. $\mu$ TAS 2007, Paris, pp. 527-529

[7] N. Sabourault et al, Organic Letters, Vol 4, No 13. (2002), pp. 2117-2119.

[8] H. Danneberg et al, J. Appl. Polym. Sci., 5, 1961, pp. $125-134$ 